J. Lake Sci. (湖泊科学), 2020, 32(3): 735-744

DOI 10. 18307/2020. 0313

(c) 2020 by Journal of Lake Sciences

\title{
环太湖地区河道和湖泊沿岸带水生植物多样性现状调查
}

\author{
谢洪民 ${ }^{1,2,3}$, 李启升 ${ }^{1,2,3}$, 刘帅领 ${ }^{4}$, 张 又 $^{3}$, 黄晓龙 ${ }^{3 * *}$, 李宽意 ${ }^{1,2,3,5}$ \\ (1: 上海海洋大学水产科学国家级实验教学示范中心, 上海 201306) \\ (2: 上海海洋大学上海水产养殖工程技术研究中心, 上海 201306) \\ (3: 中国科学院南京地理与湖泊研究所湖泊与环境国家重点实验室, 南京 210008) \\ ( 4 : 江苏江达生态环境科技有限公司, 无锡 214135) \\ (5: 中国科学院大学中丹学院, 北京 100049)
}

\begin{abstract}
摘 要: 环太湖地区经济发达, 人口密集, 近年来面临严重的富营养化、生境退化和外来水生物种人侵等环境问题. 为明 确环太湖地区河道和湖泊沿岸带水生植物多样性现状, 于 2018 年开展本地和外来水生植物多样性调查. 结果表明: (1) 在环太湖地区共计进行 65 个群落样方调查分析, 记录到 38 种水生植物, 分属 22 科 29 属; (2) 环太湖地区 31 个河道样方 中人侵沉水植物水盾草重要值最高, 其次为另一种人侵漂浮植物风眼蓝, 在 34 个湖泊沿岸带样方中风眼蓝重要值最高; (3) 环太湖地区河道和湖泊沿岸带样方生物多样性分别随着水盾草和风眼蓝盖度的增加而降低, 表明外来人侵植物影响 水生植物生物多样性. 环太湖地区湖泊需加强防范风眼蓝漂浮生长分布范围扩大和水盾草沿河道人侵太湖湖区. 这一地 区同时面对水生植物覆盖面积减少和外来植物人侵问题, 建议进行全太湖流域水生植物分布区域和生物多样性摸底普 查,加强对外来人侵植物传播的监控, 建立水生植物自然保护区.
\end{abstract}

关键词: 环太湖地区; 水生植物;生物入侵; 凤眼蓝;水盾草

\section{Investigation on the status quo of aquatic plant biodiversity in the riverways and littoral zones of the Lake Taihu rim *}

XIE Hongmin ${ }^{1,2,3}$, LI Qisheng ${ }^{1,2,3}$, LIU Shuailing ${ }^{4}$, ZHANG You ${ }^{3}$, HUANG Xiaolong ${ }^{3 * *}$ \& LI Kuanyi ${ }^{1,2,3,5}$

(1: National Demonstration Center for Experimental Fisheries Science Education, Shanghai Ocean University, Shanghai 201306 , P.R.China)

(2: Shanghai Engineering Research Center of Aquaculture, Shanghai Ocean University, Shanghai 201306, P.R.China)

(3: State Key Laboratory of Lake Science and Environment, Nanjing Institute of Geography and Limnology, Chinese Academy of Sciences, Nanjing 210008, P.R.China)

(4. Jiangsu Jiangda Eco Technology Co. Ltd., Wuxi 214135, P.R.China)

(5: Sino-Danish College, University of Chinese Academy of Sciences, Beijing 100049, P.R.China)

Abstract: The Lake Taihu rim has a robust economy and a dense population. In recent years, this area is facing severe environmental problems such as eutrophication, habitat degradation and invasion of introduced aquatic species. Aimed to clarify the status quo of aquatic plant bio-diversity in riverway and littoral zone of the Lake Taihu rim, surveys on native and introduced aquatic plants were performed in this area in 2018. The results indicate that: (1) In total, 38 aquatic plants species in 22 families and 29 genera were recorded in 65 community quadrats in the Lake Taihu rim in 2018; (2) An invasive submerged plant Cabomba caroliniana has the highest relative importance value $(R I V)$ among 31 riverway quadrats, followed by an invasive free-floating plant Eichhornia crassipes, and the latter one has the highest RIV among 34 littoral zone quadrats; (3) The aquatic plant biodiversity in-

* 2019-09-06 收稿; 2019-11-08 收修改稿.

国家自然科学基金重点项目 (31930074)、国家水体污染控制与治理科技重大专项 (2017ZX07203-005)、中国科学 院科技服务网络 STS 计划区域重点项目 (KFJ-STS-QYZD-099) 和中国科学院南京地理与湖泊研究所引进人才启动 项目 (NIGLAS2018QD01) 联合资助.

** 通信作者; E-mail:xlhuang@ niglas.ac.cn. 
dices decreased with the increasing coverage of $C$. caroliniana and E. crassipes in riverway and littoral zone quadrats, respectively, indicating that the invasive plants had an impact on the biodiversity of aquatic plants. The expanding distribution of the free-floating plant E. crassipes, and the possible invasion of the submerged plant $C$. caroliniana alongside river channels should be monitored in the Lake Taihu. Facing the problems of reducing coverage of aquatic plants and the invasion of introduced plants, it is recommended to conduct a full investigation on the distribution area and bio-diversity of aquatic plants, enhance supervisory control on the spread of introduced species, and establish natural reserves on aquatic plants in the Taihu Basin.

Keywords: Lake Taihu rim; aquatic plants; biological invasion ; Eichhornia crassipes ; Cabomba caroliniana

水生植物在淡水生态系统中处于第一营养级, 为鸟类、鱼类和底栖动物等生物提供饵料、栖息地和繁殖 场所, 也是淡水生态系统生物多样性的重要构建者 ${ }^{[1-4]}$. 目前在中国受包括水利工程、水污染、工农业用水在 内的诸多直接和间接人类活动影响, 包括水生植物在内的水生生境发生了退化 ${ }^{[5-7]}$.太湖流域湖泊水系发 达, 水源补给充沛, 开发历史悠久, 人为活动影响强烈 ${ }^{[8-9]}$. 在这一地区湖泊均为浅水湖泊, 目前大多面临富 营养化、生境退化、外来生物人侵等诸多环境问题, 其中最大湖泊为太湖, 也是我国长江中下游地区五大淡 水湖泊之一, 平均深度仅有 $1.9 \mathrm{~m}^{[10]}$. 作为城市型浅水湖泊, 太湖长期以来接纳了大量陆地面源和点源污染 物, 导致湖水水质恶化, 面临蓝藻暴发、水生植物种数减少和覆盖率下降等诸多环境问题 ${ }^{[1-12]}$.

随着水族市场日渐繁荣, 多种观赏水生植物被引人中国 ${ }^{[13]}$. 另外随着国际间贸易增加, 部分水生植物无意 中被引人,逸生后对水生生态系统有着多种影响 ${ }^{[14]}$. 生物人侵已经被当作对本地生物、群落和生态系统的严重 威胁 ${ }^{[15-16]}$,一般认为生物人侵对水生生态系统的影响要大于对陆生生态系统的影响 ${ }^{[17]}$. 目前已经有一些太湖 流域水生植物分布相关文章发表, 在太湖流域局部或大范围的野外调查都显示本地植物占据优势, 如赵凯等 2012 年在太湖贡湖湾水生植被分布调查表明本地植物竹叶眼子菜 (Potamogeton wrightii) (原文为马来眼子菜 (Potamogeton malaianus), 根据 Wiegleb 的辨析 ${ }^{[18]}$ 和 flora of China ${ }^{[19]}$, 此名称不再使用, 取而代之的是竹叶眼子 菜 (Potamogeton wrightii) ) 优势群落分布面积最广 ${ }^{[20]}$, 耿荣妹等 2014 年在太湖东部湖滨带水生植物调查表明 本地植物占据优势 ${ }^{[21]}$. 赵凯等 2014 年在太湖全湖调查结果表明本地植物竹叶眼子菜分布面积最大 ${ }^{[12]}$, 且这 一地区水生植物数量和分布面积都呈现下降趋势, 但是这些研究更多地聚焦于本地植物, 对外来植物的调查 和研究较为滞后. 通过遥感影像并结合植物生活史分析可区分不同水生植物, 但精度不高 ${ }^{[22-23]}$, 目前尚无较好 区分大面积本地植物和外来植物的方法, 传统的野外调查仍不可替代. 为明确环太湖地区河道和湖泊沿岸带 外来和本地水生植物生物多样性现状, 为这一地区水生植物保护提供数据支持, 进行了此次调查.

基于前人野外调查结果, 太湖流域主要外来人侵植物为风眼蓝 (Eichhornia crassipes, 又名凤眼莲或水葫 芦) 和喜旱莲子草 (Alternanthera philoxeroides, 又名空心莲子草或水花生 ${ }^{[20]}$. 另外一种外来植物水盾草 $(C a-$ bomba caroliniana) 近年逐渐成为沉水植物中的优势人侵种, 在 2016 年被列入第四批《中国自然生态系统外 来人侵物种名单》 ${ }^{[24]}$. 受到入湖河道建闸防汛和环太湖大堤修建的影响, 进出太湖河道多由人为控制, 太湖 已接近封闭湖泊,湖泊的水量交换周期约为 300 天 ${ }^{[25]}$. 环太湖地区水体按照水流性质可分为河道和湖泊沿 岸带两大水体类型, 本研究中河道包括河流、运河、沟渠和溪流等, 湖泊沿岸带包括湖泊、水库近水区、池塘 和沼泽等 ${ }^{[26]}$. 在太湖流域这两大水体类型间存在较大差异 ${ }^{[27-28]}$. 本研究在环太湖地区区分这两种不同类型 水体的同时, 将各采样点的水体理化数据进行比较研究, 探讨本地和外来水生植物分布和多样性现状, 为预 防和治理人侵水生植物提供可能的控制方法.

\section{1 方法}

\section{1 调查方法}

环太湖地区河道和湖泊沿岸带野外调查于 2018 年 7 月、 8 月和 10 月进行, 群落样方调查时选择典型生 境进行取样, 共计调查了 65 个群落样方 (图 1). 按照常用水生植物采样方法, 在生境随机选取面积为 $1 \mathrm{~m} \times 1$ $\mathrm{m}$ 的样方 ${ }^{[29-30]}$. 记录样方编号、采集人、水体类型、生境类型、人为干扰程度、周围地形和环境等. 使用 GPS 记 录仪记录样方经纬度和海拔高度. 原位测定使用 YSI 多参数水质分析仪测定样方内水体 $\mathrm{pH}$ 、电导率、溶解 氧等参数; 采集水样后带回实验室测定样方水体总氮和总磷浓度. 按下面的公式计算群落样方中水生植物 的 $\alpha$-多样性指数 ${ }^{[31-32]}$ : 
辛普森多样性指数 (Simpson diversity index) : $D=1-\Sigma P_{i}{ }^{2}=1-\Sigma\left(N_{i} / N\right)^{2}$

香农-威纳多样性指数 (Shannon-Wiener diversity index): $H^{\prime}=-\Sigma P_{i} \ln P_{i}$

物种均匀度指数 ( species evenness index, Pielou index) : $E=\left(-\Sigma P_{i} \ln P_{i}\right) / \ln N$

式中, $P_{i}=N_{i} / N, N_{i}$ 为第 $i$ 个群落样方中水生植物的个体数, $N$ 为群落样方中全部物种的个体数.

调查研究中采用目视估测法计算群落样方中所有物种相对盖度 $R C$ ( relative coverage) 和相对丰富度 $R A$ (relative abundance), 相对盖度 $R C=$ 样方中某种植物的投影盖度/样方中全部植物的投影盖度, 相对丰富度 $R A=$ 样方中某种植物的株数/样方中全部植物的总株数. 本研究中相对频度 $R F$ (relative frequency) $=$ 样方 中某种植物的频度/样方中所有植物的频度总和. 群落样方中物种相对优势的相对重要性指数 (relative importance value, $R I V)$ 计算公式为 ${ }^{[33-34]}$ :

\section{2 数据处理}

$$
R I V=(R C+R A+R F) / 3
$$

在统计分析之前由 Shapiro-Wilk 检验确定数据是否满足正态性假设, 由 Levene 检验确定数据是否满足 方差齐次性假设. 如果数据不能满足正态性或方差齐次性假设, 则此组数据和对应的数据均进行 $\lg (x+1)$ 转 换以满足数据正态性假设和方差齐次性假设. 为分析不同水体类型和环境因子对水生植物多样性的作用, 进行典范对应分析 (canonical correspondence analysis, CCA). 以 3 种物种 $\alpha$-多样性指数为响应变量, 以水体 $\mathrm{pH}$ 、电导率、溶解氧、总氮和总磷为解释变量, 两种水体类型 (河道和湖泊沿岸带) 作为名义变量 (dummy variables) 处理. 在分析前对 $\alpha$-多样性指数进行去趋势对应分析 (detrended correspondence analysis, DCA), 本研 究中 4 个轴的梯度长度均小于 3 , 则选用线性模型主成分分析 (principal components analysis, PCA). 典范对 应分析在 Canoco 4.5 软件 (Microcomputer Power, Ithaca, USA) 中进行. 使用线性回归模型分析外来人侵水 生植物在河道和湖泊沿岸带群落中出现的盖度和物种多样性的关系. 数据分析在 SPSS Statistics 19 软件 (IBM Corp., New York, US) 中进行. 使用 ArcGIS 10.3 软件 (Esri Inc., Redlands, US) 完成地图绘制.

\section{2 结果}

\section{1 环太湖地区河道和湖泊沿岸带水生植物多样性现状}

2018 年在环太湖地区河道和湖泊沿岸带共对 65 个群落样方进行分析 ( 图 1), 其中河道样方 31 个, 湖 泊沿岸带样方 34 个, 总计调查到 38 种水生植物, 分属 22 科 29 属, 其中水鳖科和禾本科植物最多, 各有 4 种. 本调查研究河道样方中共计 25 种水生植物 (表 1 ), 其中水盾草重要值最高 $(R I V=15.22$ ), 其次为凤眼蓝 $(R I V=11.32)$. 湖泊沿岸带样方中共计 24 种水生植物 (表 2 ), 其中凤眼蓝重要值最高 $(R I V=8.72)$, 其次为 金鱼藻 $(R I V=5.22)$.

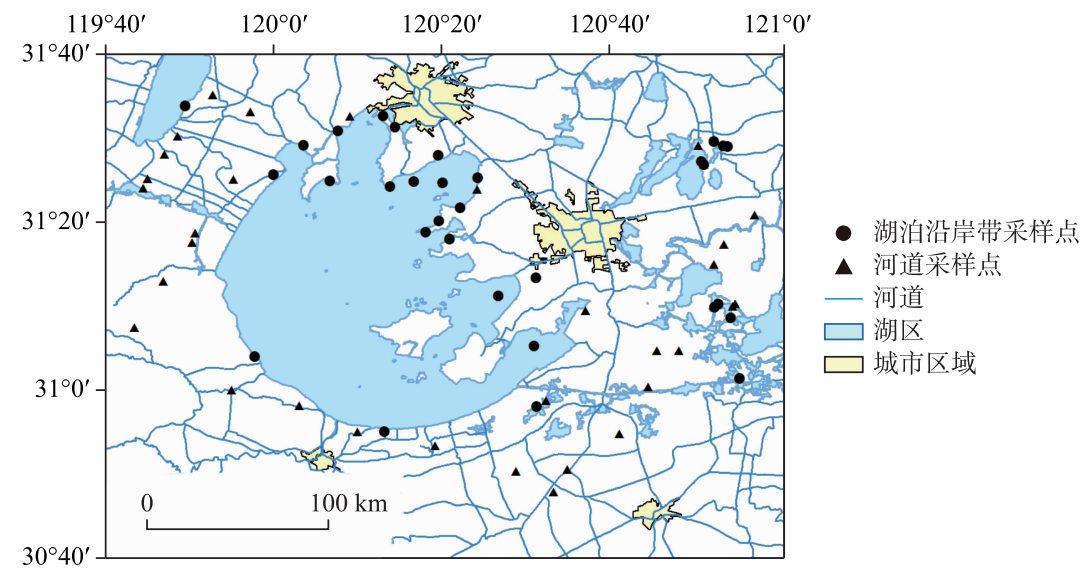

图 1 环太湖地区河道和湖泊沿岸带群落样方采样点

Fig.1 The sampling plots of plant communities in the riverways and littoral zones of the Lake Taihu rim 
表 1 环太湖地区河道水生植物相对重要性指数

Tab.1 The relative importance value of aquatic plants in the riverways of the Lake Taihu rim

\begin{tabular}{llllll}
\hline 物种 & 学名 & 重要值 & 物种 & 学名 & 重要值 \\
\hline 水盾草 & Cabomba caroliniana & 15.22 & 䔨菜 & Ipomoea aquatica & 0.61 \\
凤眼蓝 & Eichhornia crassipes & 11.32 & 红菱 & Trapa bicornis & 0.53 \\
黑藻 & Hydrilla verticillata & 8.82 & 槐叶苹 & Salvinia natans & 0.47 \\
喜早莲子草 & Alternanthera philoxeroides & 6.34 & 大薸 & Pistia stratiotes & 0.46 \\
浮萍 & Lemna minor & 5.70 & 荇菜 & Nymphoides peltata & 0.42 \\
水鳖 & Hydrocharis dubia & 4.70 & 大莰藻 & Najas marina & 0.21 \\
苦草 & Vallisneria natans & 4.02 & 丝叶眼子菜 & Stuckenia filiformis & 0.20 \\
金鱼藻 & Ceratophyllum demersum & 2.93 & 华夏慈姑 & Sagittaria trifolia subsp. leucopetala & 0.18 \\
粉绿狐尾藻 & Myriophyllum aquaticum & 2.09 & 泽泻 & Alisma plantago-aquatica & 0.11 \\
密刺苦草 & Vallisneria denseserrulata & 1.50 & 葚草 & Potamogeton crispus & 0.10 \\
竹叶眼子菜 & Potamogeton wrightii & 1.23 & 水禾 & Hygroryza aristata & 0.05 \\
穗花狐尾藻 & Myriophyllum spicatum & 1.06 & 水葱 & Schoenoplectus tabernaemontani & 0.04 \\
芦苇 & Phragmites australis & 0.68 & 梭鱼草 & Pontederia cordata & 0.03 \\
水龙 & Ludwigia adscendens & 0.66 & & & \\
\hline
\end{tabular}

表 2 环太湖地区湖泊沿岸带水生植物相对重要性指数

Tab.2 The relative importance value of aquatic plants in the littoral zones of the Lake Taihu rim

\begin{tabular}{lllllc}
\hline 物种 & 学名 & 重要值 & 物种 & 学名 & 重要值 \\
\hline 凤眼蓝 & Eichhornia crassipes & 8.72 & 李氏禾 & Leersi ahexandra & 0.93 \\
金鱼藻 & Ceratophyllum demersum & 5.22 & 穗花狐尾藻 & Myriophyllum spicatum & 0.76 \\
菱角 & Trapa japonica & 4.72 & 荷花 & Nelumbo nucifera & 0.75 \\
槐叶苹 & Salvinia natans & 3.35 & 黑藻 & Hydrilla verticillata & 0.59 \\
红菱 & Trapa bicornis & 2.39 & 苦草 & Vallisneria natans & 0.41 \\
水盾草 & Cabomba caroliniana & 1.96 & 密刺苦草 & Vallisneria denseserrulata & 0.33 \\
水龙 & Ludwigia adscendens & 1.95 & 大茨藻 & Najas marina & 0.31 \\
浮萍 & Lemna minor & 1.87 & 水鳖 & Hydrocharis dubia & 0.19 \\
细果野菱 & Trapa incisa & 1.84 & 东方香蒲 & Typha orientalis & 0.14 \\
金银莲花 & Nymphoides indica & 1.75 & 竹叶眼子菜 & Potamogeton wrightii & 0.10 \\
菰 & Zizania latifolia & 1.25 & 狭叶香蒲 & Typha angustifolia & 0.03 \\
苂实 & Euryale ferox & 1.18 & 纤细莰藻 & Najas graminea & 0.03 \\
喜早莲子草 & Alternanthera philoxeroides & 1.11 & & & \\
\hline
\end{tabular}

\section{2 环太湖地区河道和湖泊沿岸带水生植物多样性与水体指标的关系}

不同水体类型和环境因子对水生植物多样性作用的 PCA 排序分析结果表明,本研究水生植物 3 种 $\alpha$-多 样性指数中香农-威纳多样性指数和物种均匀度指数关系较为密切, 与辛普森多样性指数关系不密切 (图 2). 环境因子总氮、总磷、 $\mathrm{pH}$ 与电导率之间相互呈正相关关系, 而与采样点经度与纬度呈负相关关系 (图 2). 溶解氧除和 $\mathrm{pH}$ 、采样点经度和纬度呈正相关关系外, 与总氮、总磷、电导率均呈负相关关系 (图 2). 本研究中 将两种水体类型 (河道和湖泊沿岸带) 作为名义变量处理参与 PCA 排序, 相比河道, 湖泊沿岸带水生植物 3 种多样性指数距离较近, 表明湖泊沿岸带多样性指数平均值较高 (图 2).

\section{3 环太湖地区外来生物盖度与物种多样性的关系}

由于水盾草和凤眼蓝分别为环太湖地区河道和湖泊沿岸带水生植物优势种, 本研究按照水盾草和风眼 蓝在群落中出现的相对盖度分别得到这两种植物在环太湖地区不同类型水体的群落物种多样性关系 (图 3, 图 4). 结果表明, 在环太湖地区河道和湖泊沿岸带水生植物生物多样性指数与水盾草和风眼蓝盖度呈现负 相关趋势, 部分群落甚至成为只包含一种外来人侵物种的单物种群落 (mono-species community). 


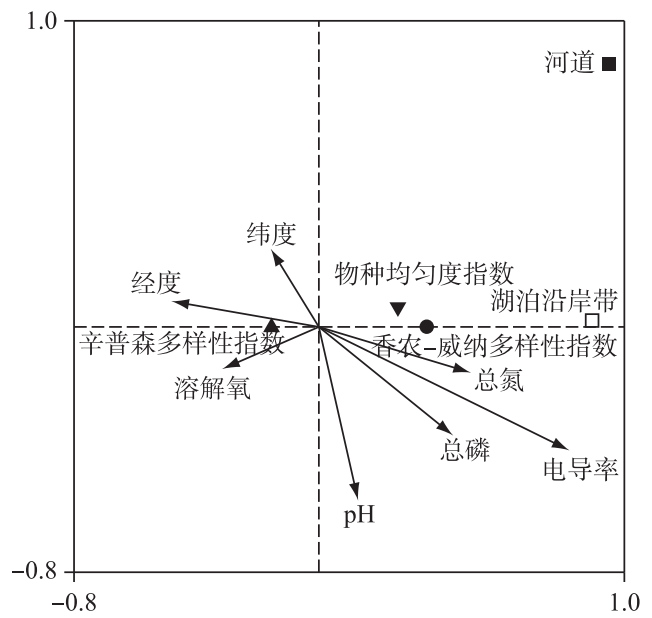

图 2 水生植物群落样方 $\alpha$-多样性指数线性模型 PCA 排序图

(不同环境因子作为解释变量用箭头表示, 两种水体类型 (河道和湖泊沿岸带) 作为名义变量处理)

Fig. 2 Linear model PCA ordination of $\alpha$-diversity indices of aquatic plant community quadrats

(Different environmental factors are indicated by arrows as explanatory variables, and different water types ( riverways and littoral zones) are treated as dummy variables)

\section{3 讨论}

之前的研究表明自 1960 年以来已有 23 种水生植物从太湖中消失 ${ }^{[12]}$, 遥感资料研究显示太湖水生植物 分布区域自 1980 年以来呈现显著下降趋势 ${ }^{[35-36]}$, 而本次调查研究结果表明目前环太湖地区水盾草和风眼 蓝分别为河道 (表 1) 和湖泊沿岸带水生植物优势种 (表 2), 并分别对水生植物生物多样性指标有影响 (图 3 , 图 4), 表明外来植物人侵已经成为这一地区水生植物管理所面临的严重问题. 本地水生植物退化和外来 水生植物人侵已经成为环太湖地区水生植物面临的两大问题,如何阻止水生植物的退化趋势和控制外来水 生植物入侵应该作为环太湖地区水生植物管理的优先课题.

生物人侵能够影响生物群落物种丰富度和多样性, 改变群落结构, 已经成为对淡水生态系统生物多样 性的严重威胁之一 ${ }^{[37-39]}$. 人侵植物通常比本地植物有更优势的性状指标, 也对变化的环境条件更具适应性, 这使得其在与本地植物的竞争中占据优势 ${ }^{[40-42]}$, 从而致使本地植物物种减少, 群落生物多样性下降, 甚至可 能导致一些狭域分布种局部灭绝, 淡水生态系统结构和功能发生不可逆转的退化. 目前长江下游湖泊面临 水域面积萎缩、生态系统碎片化和生物多样性下降等一系列生态环境问题. 人类活动如旅游设施建设直接 降低了水域面积, 修堤筑坝降低了湖泊水环境容量与湖泊对污染物的稀释吸纳能力, 农业化肥的大量使用 造成水体富营养化加剧, 首当其冲的即是本地水生植物, 而外来植物, 尤其是人侵植物对富营养化等人为污 染耐受性高, 往往能够生存并扩散成为优势物种. 太湖流域目前面临人口增长和经济发展压力, 总面积 3.69 万 $\mathrm{km}^{2}$, 仅占中国国土面积的 $1 \%, 2017$ 年总人口 6058 万人, 占全国总人口的 $4.4 \%$; GDP 共计 80815 亿元, 占 全国 GDP 的 $9.8 \%{ }^{[9]}$. 随着物质精神文化生活水平提高, 太湖流域克氏原鳌虾、大闸蟹等养殖产业蓬勃发展, 其适口性高的植物饲料有很大比例为外来植物, 而水族市场的繁荣, 较多水族箱植物被引人这一地区. 另外 水盾草在未被列人人侵植物名录之前, 也在湿地公园建设、受污染河道治理中被用作湿地修复植物广泛种 植,这可能也促进了其传播.

本研究中湖泊沿岸带的水生植物生物多样性指标平均值高于河道, 可能原因在于湖泊水体置换时间 长, 受外界干扰较少, 水生植物生长茂盛, 繁殖体和断枝较不易通过水流传播, 对于外来水生植物人侵的抵 抗性较强, 水生植物多样性较高 ${ }^{[27-28]}$; 而河道水体中水流流速较快,一般无明显分层现象, 水体置换时间短, 受外界干扰较大, 利于水生植物繁殖体和断枝传播, 导致外来水生植物人侵严重, 水生植物多样性较低. 

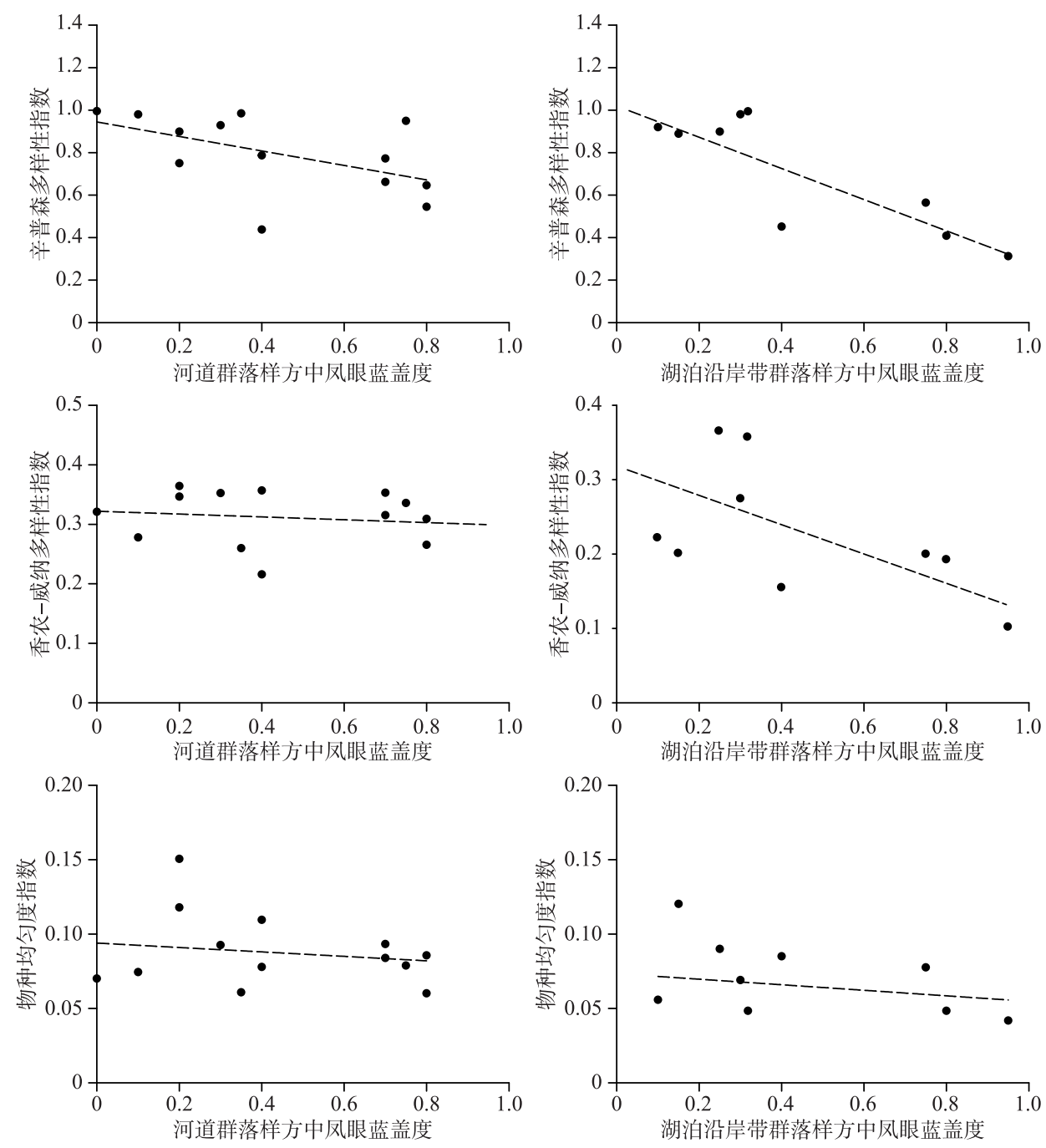

图 3 环太湖地区河道和湖泊沿岸带群落样方中凤眼蓝盖度和水生植物生物多样性指标的关系

Fig.3 Relationship between the coverage of Eichhornia crassipes and aquatic biodiversity indices of plant communities in riverways and littoral zones of the Lake Taihu rim

本次野外调查结果表明风眼蓝分别是湖泊沿岸带和河道占第 1 位和第 2 位的优势植物, 显示这种植物 已经成为环太湖地区水生态系统优势种,其植株含水量大,仅靠打捞的方法已难以将其从水体根除,使用除 草剂又会造成水体的二次污染 ${ }^{[43-44]}$. 又因为凤眼蓝不需要扎根定植生长, 并具有较高的生态耐受性和可塑 性, 可以大范围和长距离传播 ${ }^{[45-46]}$, 又有研究表明风眼蓝植株根系整体表现为一种较为特殊的辐射状聚合 构型 ( poly-herringbone branching), 这种构型根系被认为可能有助于凤眼蓝更好地适应富营养化水体环 境 ${ }^{[47]}$. 目前对风眼蓝的控制除打捞外并无较好的控制方法, 之前有研究表明冬季低温可能为限制风眼蓝生 长的关键节点 ${ }^{[48]}$, 因此除在其快速生长的夏季采取防治措施外, 还应在冬季消除沿岸带淤泥中的无性繁殖 体. 在陆生植物里选用拟南芥 (Arabidopsis thaliana) 作为模式植物 (model plant) 已开展了大量研究 ${ }^{[49]}$, 水生 植物目前还未确定模式植物, 凤眼蓝生长能力强、繁殖迅速、遗传分化低、培养容易, 并已形成全球性人侵, 是世界上影响范围最广的外来人侵水生植物 ${ }^{[43,50]}$. 已经有很多将风眼莲作为模式进行的研究工作 ${ }^{[47,51-53]}$, 在此考虑将风眼蓝作为水生植物的模式植物进行推广研究. 

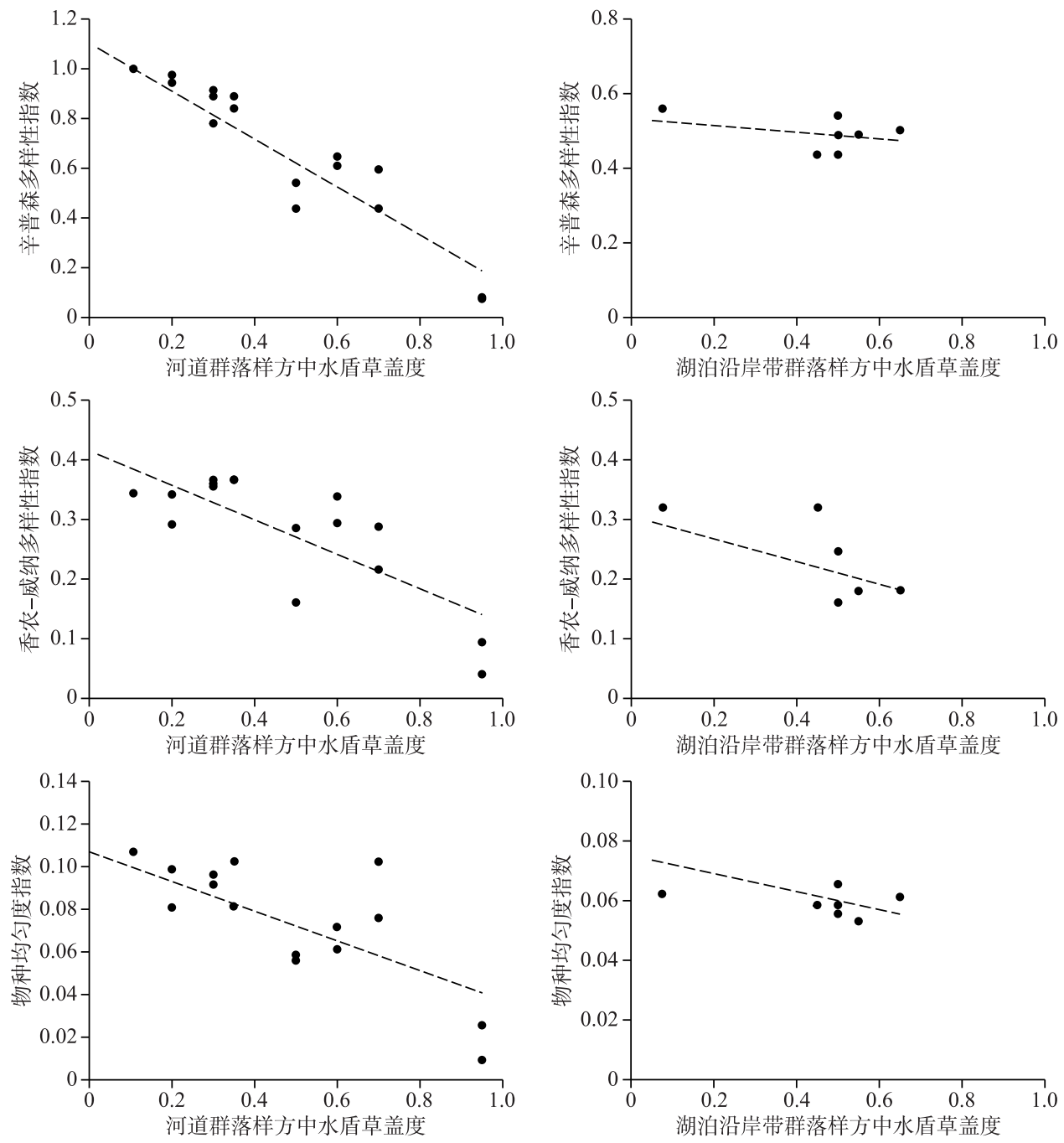

图 4 环太湖地区河道和湖泊沿岸带群落样方中水盾草盖度和水生植物生物多样性指标的关系

Fig.4 Relationship between the coverage of Cabomba caroliniana and aquatic biodiversity indices of plant communities in riverways and littoral zones of the Lake Taihu rim

水盾草约在 1980s 作为水族箱观赏植物引入中国, 由于其轮生裂叶形状独特, 易于培养, 在南方水族市 场上曾被大量出售 ${ }^{[54]}$. 中国于 1993 年在宁波首次发现野外逸生水盾草, 很快成为归化植物 (naturalized plant), 目前水盾草已经人侵中国华东和华南大片水域, 尤其以太湖流域、杭嘉湖平原、宁绍平原最为严 重 ${ }^{[55]}$. 目前水盾草在我国处于人侵早期至暴发阶段, 目前尚无较好清除措施, 需花费大量人力物力打捞, 而 在打捞过程中易形成水盾草茎叶断枝, 随水流扩散后在适宜的条件下可以定植产生新种群. 本研究中水盾 草在在河道中为占第 1 位的外来人侵植物, 而在湖泊沿岸带中分布较少, 可能是由于湖泊沿岸带相对封闭, 人侵渠道较少.

结合本次野外调查的结果, 对环太湖地区水生植物管理提出以下建议:

1) 进行全太湖流域水生植物分布区域和生物多样性摸底普查, 加强环太湖地区水生植物分布的定时监 测, 防范并遏制风眼蓝分布区域进一步扩大, 对由于水盾草过度生长形成单优群落影响到航道正常运行的 河道,加强春季打捞和并在河道清淤时对植株清除彻底; 
2) 在水盾草尚未形成优势群落,而本地沉水植物生存已经受到了抑制的河道中,要采取措施保护本地 水生植物, 在原生水生植物良好、受人为活动干扰小的地区建立水生植物自然保护区, 并加强本地沉水植物 生态修复.

致谢:感谢吴聪、何燕飞和盛天进对野外调查的支持.

\section{4 参考文献}

[ 1 ] Scheffer M ed. Ecology of shallow lakes. Dordrecht: Springer Netherlands, 2004. DOI: 10.1007/978-1-4020-3154-0.

[ 2 ] Wang H, Wang Q, Bowler P et al. Invasive aquatic plants in China. Aquatic Invasions, 2016, 11(1) : 1-9. DOI: 10. 3391/ai.2016.11.1.01.

[ 3 ] Bornette G, Puijalon S. Response of aquatic plants to abiotic factors: A review. Aquatic Sciences, 2011, 73(1): 1-14. DOI: $10.1007 / \mathrm{s} 00027-010-0162-7$.

[ 4 ] Lacoul P, Freedman B. Environmental influences on aquatic plants in freshwater ecosystems. Environmental Reviews, 2006, 14(2) : 89-136. DOI: 10.1139/a06-001.

[ 5 ] Fang JY, Wang ZH, Zhao SQ et al. Biodiversity changes in the lakes of the Central Yangtze. Frontiers in Ecology and the Environment, 2006, 4(7) : 369-377. DOI: 10.1890/1540-9295(2006)004[0369:bcitlo] 2.0.co;2.

[ 6 ] Bai X, Chen KN, Zhao HG et al. Impact of water depth and sediment type on root morphology of the submerged plant Vallisneria natans. Journal of Freshwater Ecology, 2015, 30(1) : 75-84. DOI: 10.1080/02705060.2014.970672.

[ 7 ] An SQ, Tian ZQ, Cai Y et al. Wetlands of northeast Asia and high Asia: An overview. Aquatic Sciences, 2013, 75(1): 63-71. DOI: 10.1007/s00027-012-0281-4.

[ 8 ] Wang SM, Dou HS eds. Chinese lakes. Beijing: Science Press, 1998: 5-6. [王苏民, 窦鸿身. 中国湖泊志. 北京: 科学 出版社, 1998: 5-6.]

[ 9 ] Taihu Basin Authority of Ministry of Water Resources ed. Taihu Basin \& Southeast Rivers Water Resources Bulletin, 2017. [水利部太湖流域管理局. 2017 年度太湖流域及东南诸河水资源公报, 2017.]

[10] Qin BQ, Xu PZ, Wu QL et al. Environmental issues of Lake Taihu, China. Hydrobiologia, 2007, 581(1) : 3-14. DOI: 10.1007/s10750-006-0521-5.

[11] Yang LY, Yang XY, Ren LM et al. Mechanism and control strategy of cyanobacterial bloom in Lake Taihu. J Lake Sci, 2019，31(1) : 18-27. DOI: 10.18307/2019.0102. [杨柳燕, 杨欣妍, 任丽曼等. 太湖蓝藻水华暴发机制与控制对 策. 湖泊科学, $2019, \mathbf{3 1}(1): 18-27$. ]

[12] Zhao K, Zhou YF, Jiang ZL et al. Changes of aquatic vegetation in Lake Taihu since 1960s. J Lake Sci, 2017, 29(2) : 351-362. DOI: 10.18307/2017.0211. [赵凯, 周彦锋, 蒋兆林等. 1960 年以来太湖水生植被演变. 湖泊科学, 2017, $29(2): 351-362$.

[13] Jiang H, Fan Q, Li JT et al. Naturalization of alien plants in China. Biodiversity and Conservation, 2011, 20(7): 15451556. DOI: 10.1007/s10531-011-0044-x.

[14] Hussner A, Stiers I, Verhofstad MJJM et al. Management and control methods of invasive alien freshwater aquatic plants: A review. Aquatic Botany, 2017, 136: 112-137. DOI: 10.1016/j.aquabot.2016.08.002.

[15] Goodenough A. Are the ecological impacts of alien species misrepresented? A review of the "native good, alien bad" philosophy. Community Ecology, 2010, 11(1) : 13-21. DOI: 10.1556/comec.11.2010.1.3.

[16] Luque GM, Bellard C, Bertelsmeier C et al. The 100th of the world's worst invasive alien species. Biological Invasions, 2014, 16(5) : 981-985. DOI: 10.1007/s10530-013-0561-5.

[17] Thomaz SM, Kovalenko KE, Havel JE et al. Aquatic invasive species: General trends in the literature and introduction to the special issue. Hydrobiologia, 2015, 746(1) : 1-12. DOI: 10.1007/s10750-014-2150-8.

[18] Wiegleb G. A redescription of Potamogeton wrightii (Potamogetonaceae). Plant Systematics and Evolution, 1990, 170(1/ 2) : 53-70. DOI: $10.1007 /$ bfo0937849.

[19] Flora of China Editorial Committee ed. Flora of China(Vol.23). Science Press and Missouri Botanical Garden Press, 2010: 112 .

[20] Zhao K, Li ZG, Wei HN et al. The distribution of aquatic vegetation in Gonghu Bay, Lake Taihu, 2012. J Lake Sci, 2015, 27(3) : 421-428. DOI: 10.18307/2015.0308. [赵凯, 李振国, 魏宏农等. 太湖贡湖湾水生植被分布现状 
(2012 年). 湖泊科学, 2015, 27(3): 421-428.]

[21] Geng RM, Hu XZ, Xu QJ et al. The features of aquatic macrophytes and its influent factors of lakeside zone in the east of Lake Taihu. Environmental Science \& Technology, 2016, 39(12): 17-23. [ 耿荣妹, 胡小贞, 许秋瑾等. 太湖东岸湖滨 带水生植物特征及影响因素分析. 环境科学与技术, 2016, 39(12): 17-23.]

[22] Wang Q, Zhou XD, Luo J et al. The remote sensing monitoring of dominant species of submerged vegetation of Lake Taihu with the consideration of their living histories. J Lake Sci, 2015, 27(5) : 953-961. DOI: 10.18307/2015.0523. [王琪, 周兴东, 罗菊花等. 考虑生活史的太湖沉水植物优势种遥感监测. 湖泊科学, 2015, 27 (5) : 953-961.]

[23] Zhang Z, Zhang M, Xiao W et al. Analysis of temporal and spatial variations in NDVI of aquatic vegetation in Lake Taihu. Journal of Remote Sensing, 2018, 22(2) : 324-334. [张圳, 张弥, 肖薇等. 太湖水生植被 NDVI 的时空变化特征分 析. 遥感学报, 2018, 22(2) : 324-334.

[24] Ministry of Environmental Protection of the People's Republic of China. Announcement on issuing the list of alien invasive species in China's natural ecosystem: the fourth batch, 2016. http://sts. mee. gov. cn/swaq/lygz/201708/t20170828_ 420478.shtml. [中华人民共和国环境保护部. 关于发布《中国自然生态系统外来人侵物种名单 (第四批)》的公 告, 2016.]

[25] Qin BQ, Xu H, Dong BL eds. The principle and practice of eutrophic lake restoration and management Principle and practice of eutrophic lake restoration and management. Beijing: Higher Education Press, 2011. [秦伯强, 许海, 董百丽. 富 营养化湖波治理的理论与实践. 北京: 高等教育出版社, 2011.]

[26] Lampert W, Sommer U eds. Limnoecology. Oxford: Oxford University Press, 2007: 28-29.

[27] Zhong JJ, Liu MS, Wang Y et al. Spatial correlation of major water quality indices between the lake and Rivers in Taihu Lake Basin. Chinese Journal of Ecology, 2014, 33(8) : 2176-2182. DOI: 10.13292/j.1000-4890.2014.0204. [ 钟晶晶, 刘茂松, 王玉等. 太湖流域河流与湖泊间主要水质指标的空间关联特征. 生态学杂志, 2014, 33(8): 2176-2182.]

[28] Yi J, Xu F, Gao Y et al. Variations of water quality of the major 22 inflow rivers since 2007 and impacts on Lake Taihu. $J$ Lake Sci, 2016, 28(6) : 1167-1174. DOI: 10.18307/2016.0602. [易娟, 徐枫, 高怡等. 2007 年以来环太湖 22 条主 要河流水质变化及其对太湖的影响? 湖泊科学, 2016, 28(6) : 1167-1174.]

[29] Fang JY, Wang XP, Shen ZH et al. Methods and protocols for plant community inventory. Biodiversity Science, 2009,17 (6)：533-548. [方精云, 王襄平, 沈泽吴等. 植物群落清查的主要内容、方法和技术规范. 生物多样性, 2009, 17 (6) : 533-548.]

[30] Cui XH, Chen JK, Li W. Survey methods on aquatic macrophyte vegetation in lakes in the middle and lower reaches of Changjiang River. Journal of Wuhan Botanical Research, 1999, 17(4) : 357-361. [崔心红, 陈家宽, 李伟. 长江中下游 湖泊水生植被调查方法. 武汉植物学研究, 1999, 17(4): 357-361.]

[31] Molles MC ed. Species abundance and diversity. Ecology: Concepts and applications, 7th. Ed. New York: McGraw-Hill Education, 2015: 352-371

[32] Colwell RK. III.1 biodiversity: concepts, patterns, and measurement//Levin SA, Carpenter SR, Godfray HCJ et al eds. The Princeton guide to ecology. Princeton: Princeton University Press, 2009: 257-263. DOI: 10. 1515/ 9781400833023.257.

[33] Jing ZB, Cheng JM, Su JS et al. Changes in plant community composition and soil properties under 3-decade grazing exclusion in semiarid grassland. Ecological Engineering, 2014, 64: 171-178. DOI: 10.1016/j.ecoleng.2013.12.023.

[34] Wu H, Ismail M, Ding JQ. Global warming increases the interspecific competitiveness of the invasive plant Alligator weed, Alternanthera philoxeroides. Science of the Total Environment, 2017, 575: 1415-1422. DOI: 10.1016/j. scitotenv.2016. 09.226 .

[35] Zhang YL, Qin BQ, Zhu GW et al. Profound changes in the physical environment of Lake Taihu from 25 years of long-term observations: Implications for algal bloom outbreaks and aquatic macrophyte loss. Water Resources Research, 2018, 54(7): 4319-4331. DOI: 10.1029/2017wr022401.

[36] Wang SS, Gao YN, Li Q et al. Long-term and inter-monthly dynamics of aquatic vegetation and its relation with environmental factors in Taihu Lake, China. Science of the Total Environment, 2019, 651: 367-380. DOI: 10.1016/j.scitotenv. 2018.09.216.

[37] Geist J. Integrative freshwater ecology and biodiversity conservation. Ecological Indicators, 2011, 11 (6) : 1507-1516. DOI: 10.1016/j.ecolind.2011.04.002. 
[38 ] Vilà M, Espinar JL, Hejda M et al. Ecological impacts of invasive alien plants: A meta-analysis of their effects on species, communities and ecosystems. Ecology Letters, 2011, 14(7) : 702-708. DOI: 10.1111/j.1461-0248.2011.01628.x.

[39] Reshi Z, Rasool N, Dar P et al. Impact of invasive alien plant species on aboveground and belowground species diversity in the Kashmir Himalaya, India. Invasive Plant Ecology. Boca Raton: CRC Press, 2013: 147-160. DOI: 10. 1201/ b13865-13.

[40] Richards CL, Bossdorf O, Muth NZ et al. Jack of all trades, master of some? On the role of phenotypic plasticity in plant invasions. Ecology Letters, 2006, 9( 8) : 981-993. DOI: 10.1111/j.1461-0248.2006.00950.x.

[41] van Kleunen M, Weber E, Fischer M. A meta-analysis of trait differences between invasive and non-invasive plant species. Ecology Letters, 2010, 13(2) : 235-245. DOI: 10.1111/j.1461-0248.2009.01418.x.

[42] Caplan JS, Yeakley JA. Functional morphology underlies performance differences among invasive and non-invasive ruderal Rubus species. Oecologia, 2013, 173(2) : 363-374. DOI: 10.1007/s00442-013-2639-2.

[43] IUCN. Global Invasive Species Database, 2013. http://www.iucngisd.org/gisd/100_worst.php.

[44] Li B, Liao CZ, Gao L et al. Strategic management of water hyacinth (Eichhornia crassipes), an invasive alien plant. Journal of Fudan University: Natural Science, 2004, 43(2): 267-274. [李博, 廖成章, 高雷等. 人侵植物凤眼莲管理中 的若干生态学问题. 复旦学报: 自然科学版, 2004, 43(2) : 267-274.]

[45] Chambers PA, Lacoul P, Murphy KJ et al. Global diversity of aquatic macrophytes in freshwater. Hydrobiologia, 2008, 595(1) : 9-26. DOI: 10.1007/s10750-007-9154-6.

[46] Tsuchiya T. Growth and biomass turnover of Hydrocharis dubia L. cultured under different nutrient conditions. Ecological Research, 1989, 4(2) : 157-166. DOI: 10.1007/bf02347148.

[47] Huang XL, Xu X, Liu SL et al. Impact of eutrophication on root morphological and topological performance in free-floating invasive and native plant species. Hydrobiologia, 2019, 836(1) : 123-139. DOI: 10.1007/s10750-019-3946-3.

[48] Liu JC, Chen XW, Wang YL et al. Response differences of Eichhornia crassipes to shallow submergence and drawdown with an experimental warming in winter. Aquatic Ecology, 2016, 50(2) : 307-314. DOI: 10.1007/s10452-016-9579-y.

[49] Pang PP, Meyerowitz EM. Arabidopsis thaliana: A model system for plant molecular biology. Nature Biotechnology, 1987, 5(11) : 1177-1181. DOI: 10.1038/nbt1187-1177.

[50] Zhang YY, Zhang DY, Barrett SCH. Genetic uniformity characterizes the invasive spread of water hyacinth (Eichhornia crassipes), a clonal aquatic plant. Molecular Ecology, 2010, 19(9): 1774-1786. DOI: 10.1111/j. 1365-294x. 2010. 04609.x.

[51] Wilson JR, Holst N, Rees M. Determinants and patterns of population growth in water hyacinth. Aquatic Botany, 2005,81 (1) : 51-67. DOI: 10.1016/j.aquabot.2004.11.002.

[52] Buller LS, Bergier I, Ortega E et al. Dynamic emergy valuation of water hyacinth biomass in wetlands: An ecological approach. Journal of Cleaner Production, 2013, 54: 177-187. DOI: 10.1016/j.jclepro.2013.05.006.

[53] Gamage NPD, Asaeda T. Population dynamics of water hyacinth (Eichhornia crassipes). Research report of the Research and Education Center for Inlandwater Environment Shinshu University, 2004: 35-40.

[54] He JX, Huang C, Wan FH et al. Invasion and distribution of Cabomba caroliniana in wetlands of Jiangsu, China. Chinese Journal of Applied \& Environmental Biology, 2011, 17(2) : 186-190. DOI: 10.3724/SP.J.1145.2011.00186. [何金星, 黄成, 万方浩等. 水盾草在江苏省重要湿地的人侵与分布现状. 应用与环境生物学报, 2011, 17(2): 186-190.]

[55] Yu MJ, Ding BY, Yu J et al. Basic characteristics of submerged plant communities invaded by Cabomba caroliniana and its habitat in China. Acta Phytoecologica Sinica, 2004, 28: 231-239. [于明坚,丁炳扬,俞建等. 水盾草人侵群落及其 生境特征研究. 植物生态学报, 2004, 28: 231-239.] 